\title{
Salivary psoriasin (S100A7) correlates with diffusion capacity of carbon monoxide in a large cohort of systemic sclerosis patients
}

\author{
Laura Giusti ${ }^{1 *} \mathbb{D}$, Francesca Sernissi², Elena Donadio ${ }^{1}$, Federica Ciregia ${ }^{1}$, Camillo Giacomelli², Gino Giannaccini' \\ Maria Rosa Mazzoni ${ }^{1}$, Antonio Lucacchini ${ }^{1}$ and Laura Bazzichi ${ }^{2}$
}

\begin{abstract}
Background: Systemic sclerosis (SSC) is an autoimmune disease characterized by progressive fibrosis of the skin and the internal organs. In a previous work we suggested a correlation between levels of salivary psoriasin (S100A7) and pulmonary involvement in SSC patients. The goals of this study are to determine the distribution characteristics of psoriasin in whole saliva (WS) of SSC and healthy donor populations and define its predictive value on diffusion capacity of carbon monoxide (DLCO), along with others clinical parameters.
\end{abstract}

Methods: Salivary level of psoriasin was determined by ELISA kit in 134 SSc patients, 63 Raynaud syndrome patients, 40 patients affected by other connective diseases (non-case) and 74 healthy control subjects.

Results: A significant increase of salivary psoriasin was observed in SSc patients when compared with other healthy and pathological controls. Moreover, we confirmed the efficacy of salivary psoriasin to correlate with DLCO in a large cohort of SSc patients.

Conclusions: Overall our results suggest a rapid, non invasive and low costing method which can help clinicians in the evaluation of SSc pulmonary involvement.

Keywords: Systemic sclerosis (SSc), Psoriasin, S100A7, Whole saliva, Biomarker, Diffusion capacity of carbon monoxide (DLCO)

\section{Background}

Human psoriasin (S100A7) is a small calcium binding protein which belongs to the multigenic S100 family of EF-hand proteins $[1,2]$. Human psoriasin was originally identified as an $11.4 \mathrm{kDa}$ protein upregulated in inflamed hyperplastic psoriatic skin [3] where it acts as chemoattractant for T-cells to amplify inflammatory response in psoriasis. Recently, an highly homologous (93\% of identity) protein, koebnerisin (S100A15) [4], was also identified as up-regulated in psoriasis, thereby suggesting a role in the inflammatory phenotype. Nonetheless, a recent work of Wolf and coworkers [4] reveals an unexpected

\footnotetext{
*Correspondence: laura.giusti@unipi.it

1 Department of Pharmacy, University of Pisa, Via Bonanno 6,

56126 Pisa, Italy

Full list of author information is available at the end of the article
}

biological diversity into properties and functional mechanisms of psoriasin and koebnerisin to induce inflammation suggesting a functional synergism in inflammatory diseases. In fact, despite their high homology, both antimicrobial peptides psoriasin and koebnerisin are differentially regulated by different cell types in the skin and have distinct proinflammatory functions and mechanisms of action [5]. Nonetheless they acts synergistically as "alarmins" to prime skin cells for production of immunotropic cytokines, particularly the Th17 cytokines, that further amplify the inflammatory response. On the other hand, recent researches on Th17 function have associated cytokines in the development and pathogenesis of autoimmune diseases such as SSc and rheumatoid arthritis (RA) [5]. Focusing on pulmonary manifestations of SSc disease serum levels of some cytokines, i.e. IL-17, IL-21 and IL-23, [6] and serum amyloid A [7] have been linked 
to lung involvement and have been suggested as potential biomarkers for this complication.

In a previous study using a proteomic approach, we have shown an association of salivary psoriasin with SSc clinical manifestations suggesting $12 \mathrm{kDa}$ psoriasin as a potential predictor of pulmonary involvement [8]. The novelty of our findings was the detection of marker in whole saliva (WS) whose sampling is a noninvasive, simple, safe and stress-free procedure that can be applied to large groups of subjects. Starting from preliminary results, in this work we extended the evaluation of the presence of salivary psoriasin and its correlation with clinical parameters in a large cohort of SSc patients. Therefore the aims of this study were: (1) to determine salivary psoriasin concentrations by a specific ELISA kit; (2) to define the distribution characteristics of salivary psoriasin levels in SSc patient and healthy subject populations by SPSS statistic analysis; (3) to confirm the correlation of the presence of salivary psoriasin with pulmonary involvement in the SSc population.

\section{Patients and methods}

\section{Patients}

One hundred thirty-four consecutive adult patients with diagnosis of SSc (16 male, 118 female; median age $55.4 \pm 13.1$ years), enrolled between January 2009 and June 2011, were included in the study. The study was approved by Local Ethics Committee. All patients met the ACR criteria [9]. WS samples were obtained at scheduled visits after patients signed consent form approved by Local Ethics Committee. WS was also collected from sixty-three patients with Raynaud syndrome ( 8 male, 55 female; median age $51.3 \pm 16.7$ years), forty patients affected by other connective diseases (non-case) (20 male, 20 female; median age $54.8 \pm 12.1$ years) and seventy-four healthy volunteers (14 male, 60 female; median age $40.2 \pm 20.7$ years). The clinical features of SSc patients are summarized in Table 1. Patients affected by Raynaud's syndrome ( 24 primary and 30 secondary) and noncase patients (8 Undifferentiated Connective Tissue Disease (UCTD), 2 Sjogren's syndrome, 17 psoriasis, 4 morphea, 4 polymyalgia, 1 myositis, 2 dermatomyositis, 1 rheumatoid arthritis, 1 microangiopathy), represented the pathological controls.

\section{Ethics statements}

The research was carried out according to The Code of Ethics of the World Medical Association (Declaration of Helsinki), and the author's institutional review board has approved the study. This study was approved by the Local Ethics Committee (Comitato per la Sperimentazione Clinica dei Farmaci, Azienda Ospedaliera Universitaria
Table 1 SSC patient's clinical and serological features

\begin{tabular}{ll}
\hline Features & Data (\%) \\
\hline No. of patients & 134 \\
Age, years (mean \pm SD) & $55.4 \pm 13.1$ \\
Gender (M, F) & $16 \mathrm{M}, 118 \mathrm{~F}$ \\
Diffuse & $44(38)$ \\
Limited & $71(62)$ \\
Scl-70 & $36(27)$ \\
ACA & $47(36)$ \\
FAN Hep2 & $109(83)$ \\
Acral ulcers & $28(24)$ \\
Skin & $74(87)$ \\
Rodnan skin score & $9 \pm 7.3$ \\
Pattern capillaroscopy & $87(78)$ \\
Heart involvement & $22(19)$ \\
Kidney involvement & $8(7)$ \\
DLCO <70 & $49(42)$ \\
Lung fibrosis (HRCT) & $58(47)$ \\
Pulmonary hypertension & $11(9)$ \\
\hline DLCO diffusion capacity of & \\
\hline
\end{tabular}

DLCO diffusion capacity of carbone monoxide, HRCT high resolution computed tomography

Pisana) and signed consent forms were obtained from all patients before their enrolment in the study.

\section{Sample collection and preparation}

WS samples were collected early in the morning in standard conditions, as previously described [10]. Briefly, in order to minimize protein degradation, the samples were processed immediately and kept on ice during the process. To remove debris and cells, centrifugation at $14,000 \mathrm{~g}$ for $30 \mathrm{~min}$ at $4{ }^{\circ} \mathrm{C}$ was performed and samples were stored at $-80^{\circ} \mathrm{C}$ until assayed.

\section{Salivary psoriasin assay}

The concentration of S100A7 in WS was detected by enzyme linked immunosorbent assay (ELISA) kit (CircuLex ${ }^{\mathrm{TM}}$, cat\# CY-8073) according to manufacturer's instructions. Briefly, samples (1:4000 dilution) and standards (ranging from 0.12 to $90 \mathrm{ng} / \mathrm{ml}$ ) were added in the wells. After incubation and washing, horseradish peroxidase (HRP) conjugated antibody was added, incubated and then wells were washed before the addition by substrate reagent. The reaction was stopped and the plate read at $450 \mathrm{~nm}$ using a microplate-reader Wallac Victor 1420 (PerkinElmer, San Diego, CA, USA) The limit of detection was better than $0.12 \mathrm{ng} / \mathrm{ml}$ of sample.

\section{Statistical analysis}

The normality of distribution of psoriasin levels was determined by Kolmogorov-Smirnov test. Due to 
non-normal distribution of the data, non parametric test (Mann-Whitney U test) was used to compare psoriasin levels. Spearman's rank correlations were calculated to determine the correlation between psoriasin levels and various clinical and laboratory parameters. Data were analyzed using IBM SPSS Statistics Version 19. $\mathrm{p}<0.05$ was considered statistically significant. Receiver operating characteristic (ROC) curve was performed to assess the sensitivity and specificity (IBM SPSS Statistics Version 19).

\section{Results and discussion}

The current report focuses on salivary levels of psoriasin protein in patients with SSc and their association with clinical and serological parameters, particularly with lung impairment. Pulmonary involvement is the most common manifestation of SSc. Usually, measurement of carbon monoxide diffusing capacity (DLCO), together with high-resolution computed tomography (HRTC), are initial pulmonary function tests in the evaluation of lung impairment. Nonetheless, recently Suliman and coworkers [11] have shown that the use of only pulmonary function tests as the screening method for SSc-interstitial lung disease, gives a high risk of missing significant rate of false-negative results. Previously, using a proteomic approach, we have shown several S100 proteins are altered in SSc WS including S100A7 (psoriasin) [8, 12]. In particular, we have shown that high levels of salivary psoriasin correlate with the severity of lung involvement of SSc patients. Likely, also Lakota et al. [7] have shown a correlation between high levels of serum amyloid A protein and pulmonary involvement in SSc suggesting a potential role of this protein as biomarker for this complication.

In this study, using an ELISA test, a quantification of psoriasin level in WS of SSc patients compared with that of healthy and pathological control subjects was performed. Psoriasin levels were analyzed using SPSS in different groups of subjects and a non normal distribution was observed. The mean values \pm SE were $14.1 \pm 1.3$, $25.5 \pm 2.2,19.0 \pm 3.8$ and $12.1 \pm 3.3 \mu \mathrm{g} / \mathrm{ml}$ for healthy, SSc, Raynaud and noncase groups, respectively. Figure 1 shows the scatter dot plot of psoriasin concentrations in different classes of patients. Highly significant difference $\left(\mathrm{p}<0.0001^{* * * * * *)}\right.$ of expression was observed in SSc with respect to all other groups. No significant difference in psoriasin levels were detected between two clinical subtypes [diffuse SSc (dSSc) and limited SSc (ISSc)] $(p=0.73)$ (Fig. 2). The diagnostic power of psoriasin has been also analyzed. ROC curve was calculated to evaluate the ability of psoriasin to separate healthy and SSc groups (Fig. 3). The sensitivity and specificity of psoriasin were 68 and $66 \%$, respectively and the derived area

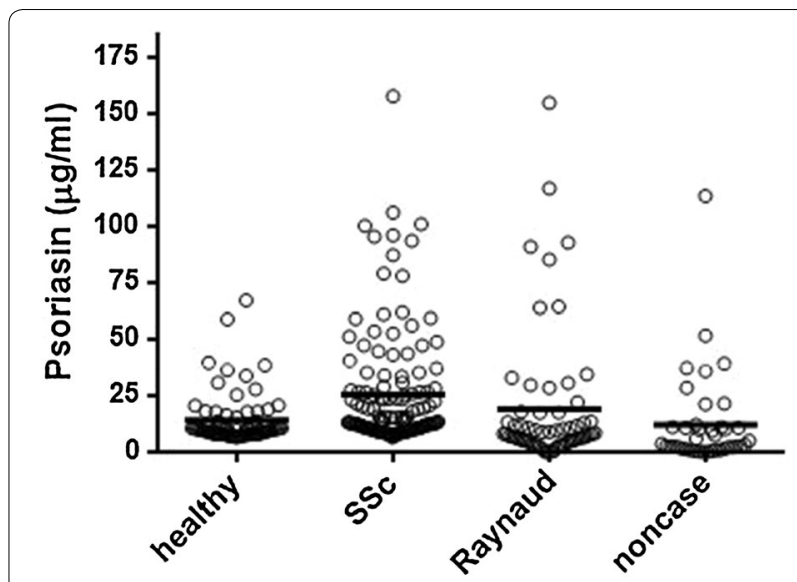

Fig. 1 Psoriasin level were determined in SSc patients, healthy subjects and pathological controls. Bold horizontal lines represent the mean value
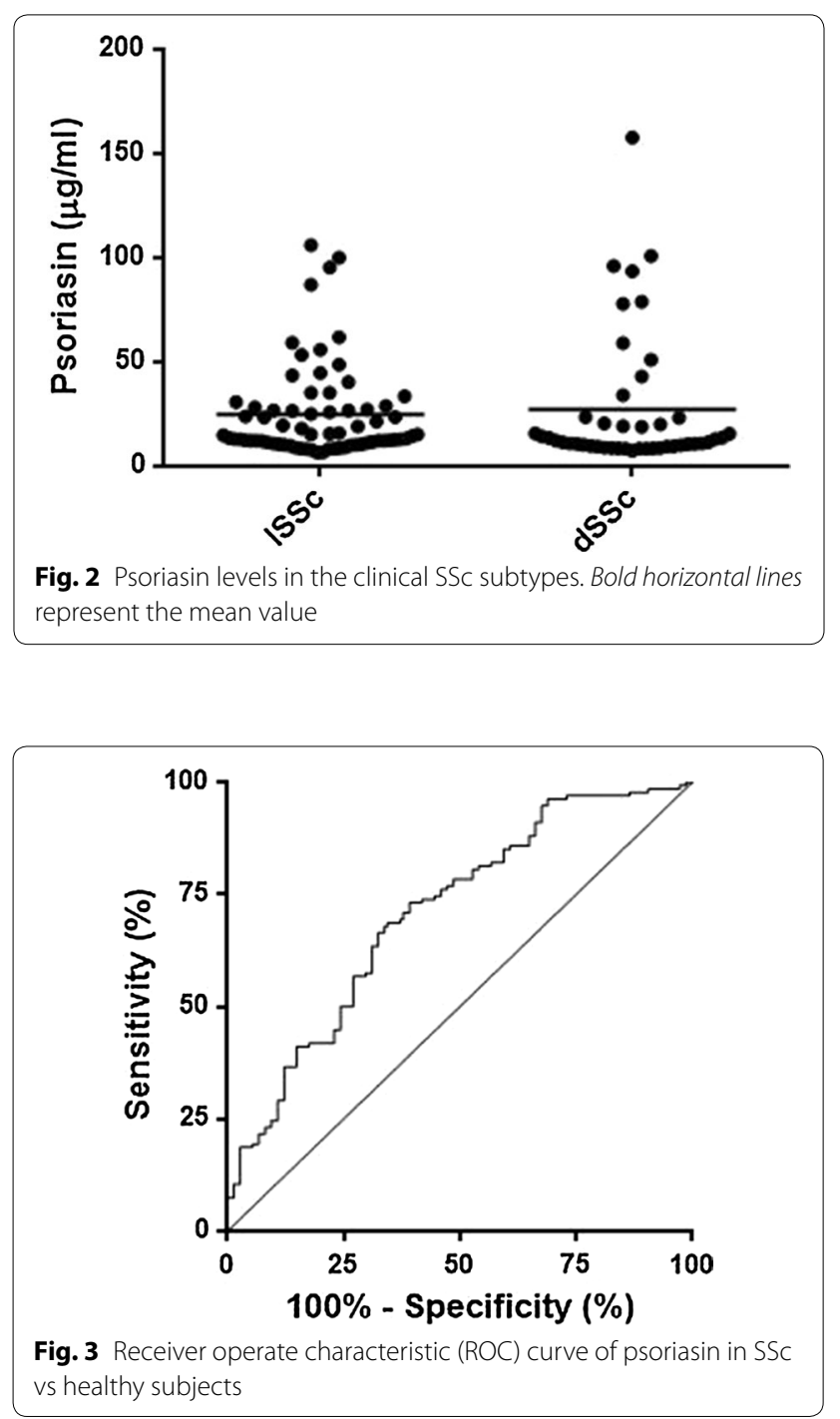
under curve (AUC) was 0.71. Moderate values of sensitivity and specificity derived from the ROC analysis performed in a large cohort of SSc patients, confirmed our previous observation that psoriasin is not suitable as early diagnostic biomarker of SSc [8]. However, Spearman's analysis showed a direct correlation of psoriasin level with the presence of serological ACA $(r=0.264$, $\left.\mathrm{p}=0.002^{* *}\right)$ and with DLCO $\left(\mathrm{r}=0.186, \mathrm{p}=0.045^{*}\right)$. No significant correlations were detected for psoriasin with other clinical and serological parameters taken in consideration (Table 2). Although we did not observe a statistically significant correlation of psoriasin levels with HRCT, however the significant correlation with DLCO, a functional pulmonary parameter, suggests that the assay of psoriasin in saliva might be a non invasive tool useful to strengthen the outcome of SSc. The pathogenetic relationship between psoriasin and lung impairment is still far from being completely clarified. As far as psoriasin is concerned different intracellular and extracellular functions have been suggested for this protein, including regulation of calcium homeostasis, cell proliferation, differentiation, apoptosis, cell invasion and motility, cytoskeleton dynamics, protein phosphorylation, regulation of transcriptional factors, immune responses, chemotaxis, antimicrobial, and inflammation [13]. In particular, a role of chemoattractant agents which are able to stimulate the neutrophyl and $\mathrm{CD} 4^{+} \mathrm{T}$ lymphocyte infiltration has been hypothesized by us to explain the link between psoriasin and lung involvement [8]. At this regard recently, the roles of Th17 and regulatory $\mathrm{T}$ (Treg) cells, in the autoimmune disease pathogenesis and in particular in SSc have been investigated [14]. In

Table 2 Clinical correlations

\begin{tabular}{|c|c|c|c|c|c|c|c|}
\hline $\begin{array}{l}\text { Non-parametric } \\
\text { correlations }\end{array}$ & $\begin{array}{l}\text { Psoriasin } \\
(\mu \mathrm{g} / \mathrm{ml})\end{array}$ & $\begin{array}{l}\text { Acral } \\
\text { ulcers }\end{array}$ & Skin & $A C A$ & SCL70 & DLCO & $\begin{array}{l}\text { Lung } \\
\text { fibrosis } \\
\text { (HRTC) }\end{array}$ \\
\hline \multicolumn{8}{|l|}{ Rho Spearman } \\
\hline \multicolumn{8}{|l|}{ Psoriasin $(\mu \mathrm{g} / \mathrm{ml})$} \\
\hline r & 1.000 & 0.032 & 0.019 & 0.264 & -0.150 & 0.186 & 0.117 \\
\hline p value & 0.0 & 0.730 & 0.860 & $0.002^{* *}$ & 0.084 & $0.045^{*}$ & 0.199 \\
\hline $\mathrm{n}$ & 134 & 121 & 90 & 133 & 133 & 117 & 122 \\
\hline \multicolumn{8}{|l|}{ Acral ulcers } \\
\hline r & 0.032 & 1.000 & 0.219 & 0.053 & 0.093 & 0.186 & 0.132 \\
\hline p value & 0.730 & 0.0 & $0.039 *$ & 0.566 & 0.311 & 0.053 & 0.161 \\
\hline$n$ & 121 & 121 & 89 & 121 & 121 & 109 & 114 \\
\hline \multicolumn{8}{|l|}{ Skin } \\
\hline r & 0.019 & 0.219 & - & -0.128 & 0.089 & 0.256 & 0.095 \\
\hline p value & 0.860 & $0.039^{*}$ & 0.0 & 0.228 & 0.406 & $0.019^{*}$ & 0.389 \\
\hline $\mathrm{n}$ & 90 & 89 & 90 & 90 & 90 & 84 & 85 \\
\hline \multicolumn{8}{|l|}{$A C A$} \\
\hline$r$ & 0.264 & 0.053 & -0.128 & 1.000 & -0.467 & -0.164 & -0.336 \\
\hline p value & $0.002^{* *}$ & 0.566 & 0.228 & 0.0 & $0.000^{* *}$ & 0.078 & $0.000^{* *}$ \\
\hline$n$ & 133 & 121 & 90 & 133 & 133 & 117 & 122 \\
\hline \multicolumn{8}{|l|}{ SCL70 } \\
\hline$r$ & -0.150 & 0.093 & 0.089 & 0.467 & 1.000 & 0.265 & 0.350 \\
\hline p value & 0.084 & 0.311 & 0.406 & $0.000^{* *}$ & 0.0 & $0.004^{* *}$ & $0.000^{* *}$ \\
\hline $\mathrm{n}$ & 133 & 121 & 90 & 133 & 133 & 117 & 122 \\
\hline \multicolumn{8}{|l|}{ DLCO } \\
\hline r & 0.186 & 0.186 & 0.256 & -0.164 & 0.265 & 1.000 & 0.451 \\
\hline p value & $0.045^{*}$ & 0.053 & $0.019^{*}$ & 0.078 & $0.004^{* *}$ & 0.0 & $0.000^{* *}$ \\
\hline $\mathrm{n}$ & 117 & 109 & 84 & 117 & 117 & 117 & 113 \\
\hline \multicolumn{8}{|c|}{ Lung fibrosis (HRTC) } \\
\hline r & 0.117 & 0.132 & 0.095 & 0.336 & 0.350 & 0.451 & 1.000 \\
\hline p value & 0.199 & 0.161 & 0.389 & $0.000^{* *}$ & $0.000^{* *}$ & $0.000^{* *}$ & 0.0 \\
\hline $\mathrm{n}$ & 122 & 114 & 85 & 122 & 122 & 113 & 122 \\
\hline
\end{tabular}

The significant correlations of psoriasin with clinical parameters are in italics

$r$ correlation coefficient, $n$ number of subjects, DLCO diffusion capacity of carbon monoxide, HRCT high resolution computed tomography

Statistical significance level: ${ }^{*} \mathrm{p}<0.05$

** $p<0.01$ 
fact, Jiang and coworkers have shown that elevated Treg (CD4, CD25 and Foxp3 positive) cells are observed in SSc patients with a high interstitial lung disease score and low DLCO value. Moreover, the authors have suggested that Th17 cells participate in both inflammation and fibrosis by secreting IL-17, which is a potent inducer of several antimicrobial peptides and proteins such as psoriasin [14]. Therefore, we reinforce our previous suggestion that the increase of psoriasin level observed in SSc patients could be linked with $\mathrm{T}$ cell abnormalities and consequent alteration in the secretion of peculiar cytokines which are leading factors SSc pathogenesis and lung impairment $[6,14]$.

Overall our results confirm the increase of psoriasin in WS of SSc patients also when compared with pathological controls and its correlation with DLCO and ACA but not with the presence of anti-Scl-70 antibodies $(\mathrm{p}=0.089)$ and lung fibrosis $(\mathrm{p}=0.199)$ as previously suggested [8].

\section{Conclusions}

The strength of our study includes the detection of marker in WS whose sampling is a noninvasive, simple, safe and stress-free procedure that can be applied to large group of subjects. On the other and the power of saliva as potential diagnostic fluid is largely documented considering numerous advantages with respect the serum [1518]. Moreover, the assay of salivary psoriasin could be a rapid method allowing clinicians to assess the pulmonary involvement, which until now is revealed only by the use of more invasive and expensive instrumental investigations. Finally, we think that a future development could be the introduction of a point of care test for the realization of a rapid, simple, low-cost and accurate method of psoriasin directly from saliva.

\begin{abstract}
Abbreviations
SSc: systemic sclerosis; WS: whole saliva; DLCO: diffusion capacity of carbon monoxide; RA: rheumatoid arthritis; UCTD: undifferentiated connective tissue disease; ELISA: enzyme linked immunoassorbent assay; HRP: horseradish peroxidase; ROC: receiver operating characteristic; HRTC: high-resolution computed tomography; AUC: area under curve; dSSc: diffuse SSc; ISSc: limited SSc.
\end{abstract}

\section{Authors' contributions}

LG, AL designed the study, coordinated the research, and with MRM analyzed data and wrote the manuscript; FS, ED and FC carried out psoriasin assay; CG and GG carried out the clinical biochemical analysis; LB carried out the clinical evaluation of the patient, participated in the design and coordination of study and helped to draft the manuscript. All authors read and approved the final manuscript.

\footnotetext{
Author details

${ }^{1}$ Department of Pharmacy, University of Pisa, Via Bonanno 6, 56126 Pisa, Italy. 2 Department of Clinical and Experimental Medicine, University of Pisa, Via Roma 67, 56126 Pisa, Italy.
}

\section{Acknowledgements}

None.

\section{Competing interests}

The authors declare that they have no competing interests.

Availability of data and materials

The data are included in the article as figures and tables.

\section{Ethics approval and consent to participate}

The study was approved by the Local Ethics Committee (Comitato per la Sperimentazione Clinica dei Farmaci, Azienda Ospedaliera Universitaria Pisana) and in accordance with Declaration of Helsinki.

\section{Funding}

This study was supported by the "Fondazione Cassa di Risparmio di Pisa."

Received: 31 May 2016 Accepted: 29 August 2016

Published online: 08 September 2016

\section{References}

1. Donato R, Cannon BR, Sorci G, Riuzzi F, Hsu K, Weber DJ, Geczy CL. Function of S100 proteins. Curr Mol Med. 2013;13:24-57.

2. Heizmann CW, Fritz G, Schäfer BW. S100 proteins: structure, functions and pathology. Front Biosci. 2002;7:d1356-68.

3. Madsen P, Rasmussen HH, Leffers H, Honoré B, Celis JE. Molecular cloning, occurrence, and expression of a novel partially secreted protein "psoriasin" that is highly up-regulated in psoriatic skin. J Invest Dermatol. 1991;97:701-12.

4. Wolf R, Ruzicka T, Yuspa SH. Novel S100A7 (psoriasin)/S100A15 (koebnerisin) subfamily: highly homologous but distinct in regulation and function. Amino Acids. 2011:41:789-96.

5. Hegyi Z, Zwicker S, Bureik D, Peric M, Koglin S, Batycka-Baran A, Prinz JC, Ruzicka T, Schauber J, Wolf R. Vitamin D analog calcipotriol suppresses the Th17 cytokine-induced proinflammatory S100"alarmins" psoriasin (S100A7) and koebnerisin (S100A15) in psoriasis. J Invest Dermatol. 2012;132:1416-24.

6. Olewicz-Gawlik A, Danczak-Pazdrowska A, Kuznar-Kaminska B, Gornowicz-Porowska J, Katulska K, Trzybulska D, Batura-Gabryel H, Silny W, Poplawski D, Hrycaj P. Interleukin-17 and interleukin-23: importance in the pathogenesis of lung impairment in patients with systemic sclerosis. Int J Rheum Dis. 2014;17:664-70.

7. Lakota K, Carns M, Podlusky S, Mrak-Poljsak K, Hinchcliff M, Lee J, Tomsic M, Sodin-Semrl S, Varga J. Serum amyloid A is a marker for pulmonary involvement in systemic sclerosis. PLoS ONE. 2015;10:e0110820.

8. Baldini C, Giusti L, Bazzichi L, Ciregia F, Giannaccini G, Giacomelli C, Doveri M, Del Rosso M, Bombardieri S, Lucacchini A. Association of psoriasin (S100A7) with clinical manifestations of systemic sclerosis: is its presence in whole saliva a potential predictor of pulmonary involvement? J Rheumatol. 2008:35:1820-4.

9. van den Hoogen F, Khanna D, Fransen J, Johnson SR, Baron M, Tyndall A, Matucci-Cerinic M, Naden RP, Medsger TA Jr, Carreira PE, Riemekasten G, Clements PJ, Denton CP, Distler O, Allanore Y, Furst DE, Gabrielli A, Mayes MD, van Laar JM, Seibold JR, Czirjak L, Steen VD, Inanc M, Kowal-Bielecka O, Müller-Ladner U, Valentini G, Veale DJ, Vonk MC, Walker UA, Chung L, Collier DH, Csuka ME, Fessler BJ, Guiducci S, Herrick A, Hsu VM, Jimenez S, Kahaleh B, Merkel PA, Sierakowski S, Silver RM, Simms RW, Varga J, Pope JE. 2013 classification criteria for systemic sclerosis: an American College of Rheumatology/European League against Rheumatism collaborative initiative. Arthritis Rheum. 2013;65:2737-47.

10. Giusti L, Baldini C, Bazzichi L, Ciregia F, Tonazzini I, Mascia G, Giannaccini G, Bombardieri S, Lucacchini A. Proteome analysis of whole saliva: a new tool for rheumatic diseases - the example of Sjögren's syndrome. Proteomics. 2007;7:1634-43.

11. Suliman YA, Dobrota R, Huscher D, Nguyen-Kim TD, Maurer B, Jordan S, Speich R, Frauenfelder T, Distler O. Brief report: pulmonary function tests: high rate of false-negative results in the early detection and screening of scleroderma-related interstitial lung disease. Arthritis Rheumatol. 2015;67:3256-61.

12. Giusti L, Bazzichi L, Baldini C, Ciregia F, Mascia G, Giannaccini G, Del Rosso M, Bombardieri S, Lucacchini A. Specific proteins identified in whole saliva from patients with diffuse systemic sclerosis. J Rheumatol. 2007:34:2063-9. 
13. Jia J, Duan Q, Guo J, Zheng Y. Psoriasin, a multifunctional player in different diseases. Curr Protein Pept Sci. 2014;15:836-42.

14. Jiang N, Li M, Zeng X. Correlation of Th17 cells and CD4 $4^{+} \mathrm{CD} 25^{+}$regulatory T cells with clinical parameters in patients with systemic sclerosis. Chin Med J (Engl). 2014;127:3557-61.

15. Amado FM, Ferreira RP, Vitorino R. One decade of salivary proteomics: current approaches and outstanding challenges. Clin Biochem. 2013:46:506-17.

16. Ciregia F, Giusti L, Da Valle Y, Donadio E, Consensi A, Giacomelli C, Sernissi F, Scarpellini P, Maggi F, Lucacchini A, Bazzichi L. A multidisciplinary approach to study a couple of monozygotic twins discordant for the chronic fatigue syndrome: a focus on potential salivary biomarkers. J Transl Med. 2013;11:243.
17. Baldini C, Giusti L, Ciregia F, Da Valle Y, Giacomelli C, Donadio E, Ferro F, Galimberti S, Donati V, Bazzichi L, Bombardieri S, Lucacchini A. Correspondence between salivary proteomic pattern and clinical course in primary Sjögren syndrome and non-Hodgkin's lymphoma: a case report. J Transl Med. 2011;9:188.

18. Bazzichi L, Ciregia F, Giusti L, Baldini C, Giannaccini G, Giacomelli C, Sernissi F, Bombardieri S, Lucacchini A. Detection of potential markers of primary fibromyalgia syndrome in human saliva. Proteom Clin Appl. 2009;3:1296-304.

\section{Submit your next manuscript to BioMed Central and we will help you at every step:}

- We accept pre-submission inquiries

- Our selector tool helps you to find the most relevant journal

- We provide round the clock customer support

- Convenient online submission

- Thorough peer review

- Inclusion in PubMed and all major indexing services

- Maximum visibility for your research

Submit your manuscript at www.biomedcentral.com/submit 